\title{
Deep learning black hole metrics from shear viscosity
}

\author{
Yu-Kun Yan $\odot,{ }^{1,2, *}$ Shao-Feng Wu$\odot,{ }^{1,3, \dagger}$ Xian-Hui Ge, ${ }^{1,3, *}$ and Yu Tian ${ }^{2,4,5,3,8}$ \\ ${ }^{1}$ Department of Physics, Shanghai University, Shanghai, 200444, China \\ ${ }^{2}$ School of Physics, University of Chinese Academy of Sciences, Beijing, 100049, China \\ ${ }^{3}$ Center for Gravitation and Cosmology, Yangzhou University, Yangzhou 225009, China \\ ${ }^{4}$ Institute of Theoretical Physics, Chinese Academy of Sciences, Beijing 100190, China \\ ${ }^{5}$ Center for Theoretical Physics, Massachusetts Institute of Technology, \\ Cambridge, Massachusetts 02139, USA
}

(Received 20 June 2020; accepted 21 October 2020; published 12 November 2020)

\begin{abstract}
Based on AdS/CFT correspondence, we build a deep neural network to learn black hole metrics from the complex frequency-dependent shear viscosity. The network architecture provides a discretized representation of the holographic renormalization group flow of the shear viscosity and can be applied to a large class of strongly coupled field theories. Given the existence of the horizon and guided by the smoothness of spacetime, we show that Schwarzschild and Reissner-Nordström metrics can be learned accurately. Moreover, we illustrate that the generalization ability of the deep neural network can be excellent, which indicates that by using the black hole spacetime as a hidden data structure, a wide spectrum of the shear viscosity can be generated from a narrow frequency range. These results are further generalized to an Einstein-Maxwell-dilaton black hole. Our work might not only suggest a data-driven way to study holographic transports but also shed some light on holographic duality and deep learning.
\end{abstract}

DOI: 10.1103/PhysRevD.102.101902

\section{INTRODUCTION}

The renormalization group (RG) is a physical scheme to understand various emergent phenomena in the world through iterative coarse graining [1-4]. Deep learning (DL) is the core algorithm of the recent wave of artificial intelligence [5]. It has been suggested that RG and DL might have a logic in common [6,7], and their relation has attracted a lot of interest [8-17].

RG is believed to be one of the key elements to understand quantum gravity. In particular, by anti-de Sitter/conformal field theory (AdS/CFT) correspondence [18-21], a strongly coupled quantum critical theory in the d-dimensional spacetime is reorganized along the RG scale, inducing a classical theory of gravity in the $(d+1)$ dimensional AdS spacetime. RG is not the only connection between DL and gravity. Through the study of tensor networks [22-24], especially the multiscale entanglement renormalization ansatz [25], it has been realized that the way the geometry is emergent from field theories usually

\footnotetext{
*ykyan.phys@gmail.com

sfwu@shu.edu.cn

*gexh@shu.edu.cn

\$ytian@ucas.ac.cn
}

Published by the American Physical Society under the terms of the Creative Commons Attribution 4.0 International license. Further distribution of this work must maintain attribution to the author(s) and the published article's title, journal citation, and DOI. Funded by SCOAP. involves the network and optimization, which are two important ingredients of DL.

Because of these connections, the deep neural network (DNN) may be capable of providing a research platform for holographic duality [26-33]. One can expect at least two benefits: It is helpful to understand how the spacetime emerges and can be used to build a data-driven phenomenological model for strongly coupled field theories. The latter was initiated in [28], where the inverse problem of the AdS/CFT is studied, that is, how to reconstruct the spacetime metric from the given field theory data by the DNN which implements the AdS/CFT. Subsequently, the so-called AdS/DL correspondence is applied to learn the bulk metric from the lattice QCD data of the finitetemperature chiral condensate. Interestingly, the emergent metric exhibits both a black hole horizon and an IR wall with finite height, signaling the crossover of QCD thermal phases [29].

Let us briefly introduce the prototype of AdS/DL (i.e., the first numerical experiment in [28]). It establishes the architecture of the DNN according to the discretized equation of motion of the $\phi^{4}$ theory minimally coupled to gravity. The training data are the one-point function and the conjugate source with a label determined by the nearhorizon scalar field. The target of learning is the metric of the Schwarzschild black hole. A key technique is to design the regularization by which the emergent metric is favored to be smooth. It is found that the DNN performs better near the boundary than near the horizon where the relative error is around $30 \%$. In [33], the AdS/DL has been used to learn 
the Reissner-Nordström (RN) metric, but the mean square error (MSE) ranges from $\mathcal{O}\left(10^{-3}\right)$ to $\mathcal{O}\left(10^{-1}\right)$. Importantly, it is revealed that the form of the regularization term must be fine-tuned for different metrics. This suggests that their DNN may not find the target metric if it was unknown previously, since it is difficult to judge which is closer to the target metric under different regularizations.

In this paper, we will extend the physical range of the AdS/DL nontrivially and illustrate that it can be realized without previous technical problems. Our strategies are as follows. First, AdS/CFT is almost customized for the computation of the transports of strongly coupled quantum critical systems at finite temperatures [34]. In particular, the application of holography is anchored partially in the prediction of the nearly perfect fluidity [35], which has been observed in the hot quark gluon plasmas and cold unitary Fermi gases [36]. With these in mind, we adapt the complex frequency-dependent shear viscosity as the given field theory data. Second, we propose to build the DNN according to the holographic RG flow of the shear viscosity. Up to the holographic renormalization, this flow was presented in the well-known holographic membrane paradigm [37,38], which interpolates the standard AdS/ CFT correspondence and the classical black hole membrane paradigm smoothly $[39,40]$. Third, we assume the existence of the horizon, which will reduce the learning difficulties. Fourth, the system error in [28] comes from adding labels on the data and introducing the regularization. Because the horizon value of the shear response is completely determined by the regularity analysis on the horizon, we can generate the data by the flow from IR to UV. The direction of information transfer is contrary to $[28,29]$, and there is no error caused by the labels of the data. Fifth, we still use the regularization to guide the network to find a smooth metric. However, our training process has two stages and the regularization is only required in the first stage, so we can choose any regularization term as long as it leads to a smaller loss in the second stage. Finally, we will discuss possible extensions and physical implications.

\section{FROM RG FLOW TO DNN}

Suppose that a strongly coupled field theory is dual to the $(3+1)$-dimensional Einstein gravity minimally coupled with matter, which admits a homogeneous and isotropic (along the field theory directions) black hole solution with the metric ansatz

$$
d s^{2}=-g_{t t}(r) d t^{2}+g_{r r}(r) d r^{2}+g_{x x}(r) d \vec{x}^{2} .
$$

When the black hole is perturbed by time-dependent sources, the shear mode $(\delta g)_{x_{2}}^{x_{1}}=h(r) e^{-i \omega t}$ of the gravitational wave is controlled by the equation of motion

$$
\frac{1}{\sqrt{-g}} \partial_{r}\left(\sqrt{-g} g^{r r} \partial_{r} h\right)+g^{t t} \omega^{2} h=0
$$

provided that the graviton is massless ${ }^{1}$ [41]. In the Hamiltonian form, the wave equation can be written as

$$
\begin{gathered}
\Pi=-\sqrt{-g} g^{r r} \partial_{r} h, \\
\partial_{r} \Pi=\sqrt{-g} g^{r r} g^{t t} \omega^{2} h,
\end{gathered}
$$

where $\Pi$ is the momentum conjugate to the field $h$. Consider the foliation in the $r$ direction and define the shear response function $\chi=\Pi /(i \omega h)$ on each cutoff surface. Substituting Eq. (3) into Eq. (4), one can obtain

$$
\partial_{r} \chi-i \omega \sqrt{\frac{g_{r r}}{g_{t t}}}\left(\frac{\chi^{2}}{g_{x x}}-g_{x x}\right)=0 .
$$

Note that this radial flow equation has been derived in [37] where the dc limit is focused on. ${ }^{2}$ We will study the frequency-dependent behavior.

Applying the regularity of $\chi$ on the horizon, one can read off the horizon value of $\chi$ directly

$$
\chi\left(r_{h}\right)=g_{x x}\left(r_{h}\right)
$$

where $r_{h}$ is the horizon radius. Taking Eq. (6) as the IR boundary condition, the flow equation can be integrated to the UV boundary. However, it should be pointed out that the response function $\chi$ on the UV boundary is not equal to the shear viscosity $\eta$ of the boundary field theory. In the Supplemental Material [45], we will clarify the relation between them using the Kubo formula of the complex frequency-dependent shear viscosity $[46,47]$ and the holographic renormalization $[48,49]$. It can be found that for a large class of holographic models, including the EinsteinMaxwell theory which will be studied below, the relation is

$$
\eta(\omega)=\chi(\omega, r)+\left.i \omega r\right|_{r \rightarrow \infty} .
$$

In the metric ansatz (1), $g_{x x}$ can be fixed as $r^{2}$ without loss of generality but $g_{t t}$ and $g_{r r}$ are independent in general. However, there are some black holes which share the feature $g_{t t} g_{r r}=1$, indicating that the radial pressure is the negative of the energy density [50]. For simplicity, we will consider this situation first and return to the more general case later. Thus, the metric ansatz can be reduced to

\footnotetext{
${ }^{1}$ The gauged coordinate-invariance symmetries in the bulk demand that the gravitons are massless and they are dual to the global spacetime symmetries on the boundary [34].

${ }^{2}$ In the Wilsonian formulation, the flow equation can be retrieved as the $\beta$ functions of double-trace couplings [42-44].
} 


$$
d s^{2}=\frac{1}{z^{2}}\left[-f(z) d t^{2}+\frac{1}{f(z)} d z^{2}+d \vec{x}^{2}\right]
$$

where we have used the coordinate $z=r_{h} / r$ so that the horizon is located at $z=1$ and the boundary at $z=0$. Accordingly, Eq. (5) can be rewritten as

$$
\left(\eta-\frac{i \omega}{z}\right)^{\prime}+\frac{i \omega}{f}\left[z^{2}\left(\eta-\frac{i \omega}{z}\right)^{2}-\frac{1}{z^{2}}\right]=0,
$$

where we have set $r_{h}=1$ and the prime denotes the derivative with respect to $z$. Note that we have replaced $\chi(\omega, z)$ with $\eta(\omega, z)-i \omega / z$ from IR to UV. Compared to Eq. (7) where the replacement occurs only on the UV, we have found that this technique reduces the discretized error considerably. The radially varying function $\eta(\omega, z)$ can be referred to as the holographic RG flow of the shear viscosity. In the following, we will build a DNN according to the flow equation (9).

Figure 1 is a schematic diagram of the DNN. The $N$ deep layers are located by discretizing the radial direction

$$
z(n)=z_{b}+n \Delta z, \quad \Delta z=\frac{z_{h}-z_{b}}{N},
$$

where $z_{b}\left(z_{h}\right)$ is the UV (IR) cutoff and the integer $n$ belongs to $[1, N]$. The trainable weights of the DNN represent the discretized metrics. The input is $\eta\left(\omega, z_{h}\right)$ and the output is $\eta\left(\omega, z_{b}\right)$. The information is transferred from the $N$ th layer (IR) to the first layer (UV). The propagation rule of $\eta(\omega, z)$ between layers is determined by the discretized representation of Eq. (9):

$$
\begin{aligned}
\operatorname{Re} \eta(z+\Delta z)= & \operatorname{Re}(z)\left[1+\Delta z \frac{2 \omega z^{2}}{f(z)}\left(\operatorname{Im} \eta(z)-\frac{\omega}{z}\right)\right] \\
\operatorname{Im} \eta(z+\Delta z)= & \operatorname{Im} \eta(z)+\Delta z \frac{\omega z^{2}}{f(z)}\left[\frac{1-f(z)}{z^{4}}\right. \\
& \left.-(\operatorname{Re} \eta(z))^{2}+\left(\operatorname{Im} \eta(z)-\frac{\omega}{z}\right)^{2}\right] .
\end{aligned}
$$

Here we have separated the discretized flow equation into real and imaginary parts for the convenience in DL.

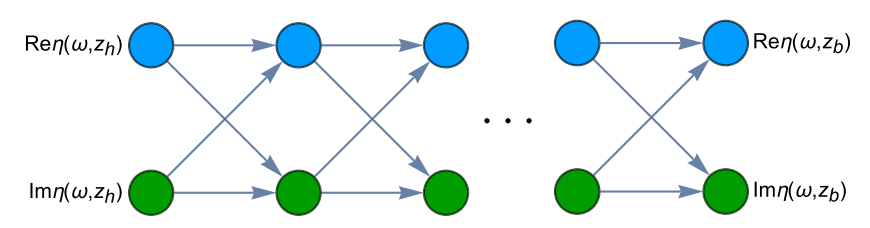

FIG. 1. The architecture of the DNN. The green and blue nodes have $N$ layers, which propagate the shear viscosity from IR to UV by discretized RG flow equations (11). The arrows indicate the direction of information transfer.
The loss function we choose is the $L^{2}$ norm

$$
L_{\mathrm{DNN}}=\sum_{\text {data }}\left|\eta\left(\omega, z_{b}\right)-\bar{\eta}\left(\omega, z_{b}\right)\right|^{2}
$$

up to a regularization term, if existent. Here, $\eta$ represents the input data and $\bar{\eta}$ is what the DNN generates.

We need a regularization term which can guide the DNN to find a smooth black hole metric. In principle, the form of the regularization term can be arbitrary as long as it can reduce the final loss. In practice, our regularization term is specified as

$$
\begin{aligned}
L_{\mathrm{REG}}= & c_{1} \sum_{n=1}^{N-1} \frac{1}{z(n)^{c_{2}}}[f(z(n+1))-f(z(n))]^{2} \\
& +c_{3}[f(z(N))-0]^{2},
\end{aligned}
$$

where the two parts are designed for the smoothness of the metric and the existence of the horizon, respectively. Three hyperparameters $c_{1}, c_{2}$, and $c_{3}$ are introduced.

\section{GENERATED DATA AND DISCRETIZED ERROR}

We specify the discretized RG flow and hence the DNN by setting $z_{b}=0.01, z_{h}=0.99$, and $N=10$. Using the discretized flow equation (11) with the IR boundary condition $\eta\left(\omega, z_{h}\right)=1+i \omega$ and the target metric, we can generate the data required by DL. Here we consider the two most famous black holes, i.e., the Schwarzschild and RN. They are characterized, respectively, by the functions

$$
\begin{gathered}
f(z)=1-z^{3}, \\
f(z)=1-z^{3}-\frac{q^{2} z^{3}}{4}+\frac{q^{2} z^{4}}{4},
\end{gathered}
$$

where $q$ is the charge density. We generate 2000 data $\left[\omega, \eta\left(\omega, z_{b}\right)\right]$ with even frequency spacing. The training set and validation set account for $90 \%$ and $10 \%$, respectively. In Fig. (2), we compare the data with the ones generated by numerically solving the continuous flow equation of the shear viscosity. It is found that the discretized error is small when the target is the Schwarzschild metric, and increases with the frequency and the charge density. The discretized error can be reduced by adding more layers but it requires powerful computing capabilities. As a proof of principle, here we simply assume that the discretized error does not affect our results qualitatively. ${ }^{3}$

\footnotetext{
${ }^{3}$ Recently, the discretized error has been taken into account in the application of AdS/DL to the QCD experimental data [51].
} 


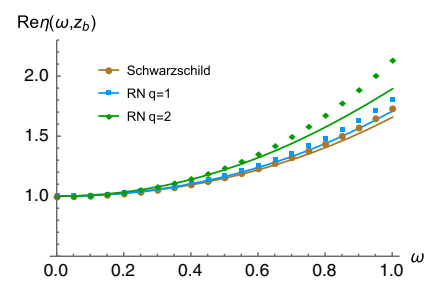

(a)

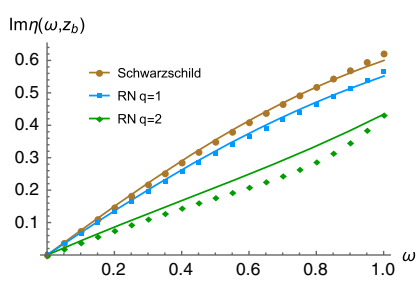

(b)

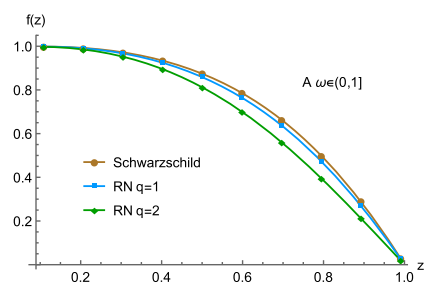

(a)

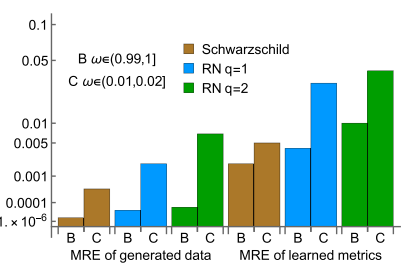

(b)

FIG. 2. The data of the shear viscosity generated by different metrics. (a) and (b) denote the real and imaginary parts, respectively. The curves are generated by the continuous holographic RG flow equation of the shear viscosity, while the markers are generated by the discretized equation.

\section{EMERGENCE AND GENERALIZATION}

With the data in hand, we will train the DNN and extract the weights. The training scheme will be given in the Supplemental Material [45]. In Table S.1 of the Supplemental Material [45], we list the training reports after two training stages of various numerical experiments. Among others, it is shown that from the dataset with $\omega \in(0,1]{ }^{4}$ the Schwarzschild and RN metrics can be learned with high accuracy: The mean relative error (MRE) is around $0.1 \%$. Note that the MSE is $\mathcal{O}\left(10^{-7}\right)$. The target and learned metrics have been plotted in Fig. 3(a).

Hereto, we almost naively select the frequency range of the data as $\Delta \omega=1$. One important question in DL is how well the model generalizes. To proceed, we consider different datasets with the narrow frequency range $\Delta \omega=$ $10^{-2}$ and keep each of them with 2000 data. Interestingly, we find that both Schwarzschild and RN metrics still can be well learned, although the error will increase when the frequency window is close to zero and especially when the charge density is large. This is shown by the MRE of the metrics learned from two typical windows; see the right half of Fig. 3(b). Furthermore, it suggests that the generalization ability of the DNN can be excellent. Indeed, in the left half of Fig. 3(b), we illustrate that using the metric learned from the data with $\Delta \omega=10^{-2}$, one can generate the data with $\Delta \omega=1$ very accurately. In the bestperforming example, the MRE of the generated data can be $\mathcal{O}\left(10^{-6}\right)$. We also note that the examples with relatively large errors in Fig. 3(b) can be expected because the dc limit of the shear viscosity is determined solely by the physics on the horizon. In particular, when the charge density increases, the RN black hole approaches extremality, and the IR CFT associated with the $\mathrm{AdS}_{2} \times \mathrm{R}^{2}$ geometry gradually begins to dominate the low-frequency physics [52,53]. Similarly, we do not expect that the DNN can learn well from a very high-frequency window, where

\footnotetext{
${ }^{4}$ The frequency is measured in units of the horizon radius.
}

FIG. 3. The performance of the DNN. (a) The curves are the target metrics, and the markers are the results learned from the data $\mathrm{A}$, which has a wide frequency range. (b) The right half of the bars represents the MRE of the metrics which are learned from the data with narrow ranges $B$ and $C$. The left half represents the MRE of the wideband data, which are generated using the metrics learned from the narrow band.

the UV CFT associated with the AdS boundary should dominate. $^{5}$

\section{BEYOND EINSTEIN-MAXWELL}

Our DNN is not only applicable to the Einstein-Maxwell theory. In the SM, we demonstrate in general that the DNN can be applied to the Einstein-Maxwell-dilaton (EMD) theory with the typical potential and coupling of the dilaton [56]. The only nontrivial constraint is that the conformal dimension of the dilaton operator $\Delta_{\phi}$ should be less than $5 / 2$. To be more specific, we further study a concrete EMD theory. It admits the analytical black hole solution with $\Delta_{\phi}=2$, and its holographic renormalization has been given in [57]. This exemplifies our general argument explicitly. We also carry out the numerical experiments as before. It is found that the performance of the DNN for the EMD black hole is similar to that for the RN; see Table S.1 in the Supplemental Material [45]. Note that at the zero-temperature limit, the EMD black hole becomes a special hyperscaling violating Lifshitz geometry with the asymptotic AdS. Moreover, here the metric components $g_{t t}(r) g_{r r}(r) \neq 1$, so what the DNN has learned is the joint factor

$$
f(z)=\left.\frac{1}{r^{2}} \sqrt{\frac{g_{t t}(r)}{g_{r r}(r)}}\right|_{r=r_{h} / z},
$$

as we will explain below.

\section{VISCOSITY AND ENTANGLEMENT}

A more general metric like the EMD black hole has two independent components $g_{t t}$ and $g_{r r}$. From Eq. (5), one can find that they appear in the form of the joint factor $g_{r r} / g_{t t}$. Therefore, without the constraint on the energy-momentum

\footnotetext{
${ }^{5}$ In fact, it has been observed in $[54,55]$ that the retarded Green function for the shear stress operator at the infinite frequency is determined by the energy density. We thank Matteo Baggioli for the discussion on this point.
} 
tensor for $g_{t t} g_{r r}=1$, the DNN still can be applied to learn the joint factor, but in general each of them cannot be learned separately from the shear response. Nevertheless, if there is another way to determine one component, the other can be obtained by the DNN. For example, there is evidence that the entanglement plays an important role in weaving the spacetime [58-62]. Among others, it has been shown that the holographic entanglement entropy $S(l)$ can be used to fix the bulk metric wherever the extremal surface reaches [63], which can be described as

$$
\left.r \sqrt{g_{r r}(r)}\right|_{r=r_{h} / z}=\frac{1}{2 \pi^{2} L} z^{2} \partial_{z} \int_{z_{b}}^{z} \frac{z_{*} S\left(z_{*}\right)}{\sqrt{z^{4}-z_{*}^{4}}} d z_{*},
$$

where we have set the gravitational constant $16 \pi G=1$, and $z_{*}$ is determined by $S^{\prime}(l)=8 \pi L / z_{*}^{2}$. Note that $l$ and $L$ denote the finite width and the (regularized) infinite length of the rectangle on which the extremal surface is anchored [59]. Since the holographic entanglement entropy is only related to $g_{r r}$, it can complement the shear viscosity to determine two metric components.

\section{CONCLUSION AND DISCUSSION}

Using a simple DL algorithm, we studied an inverse problem of AdS/CFT: Given the complex frequencydependent shear viscosity of boundary field theories at finite temperatures, can the bulk metrics of black holes be extracted? We showed that Schwarzschild, RN, and EMD metrics can be learned by the DNN with high accuracy. The network architecture can be taken as a discretized representation of the holographic RG flow of the shear viscosity, hence, supporting the underlying relationship among DL, $\mathrm{RG}$, and gravity. We pointed out that our DNN is applicable to any strongly coupled field theory provided that it is dual to the $(3+1)$-dimensional Einstein gravity minimally coupled with matter, it allows a homogeneous and isotropic black hole solution, the graviton mass in the wave equation vanishes, and the UV relation (7) holds. The extensions to the symmetry-breaking situations, the higher spacetime dimensions, and the modified theories of gravity should be worthwhile. Among others, we note that using the wave equation with the graviton mass which has been built in [41], one can construct the RG flow and the DNN where the graviton mass is encoded into new trainable weights. It is interesting to study whether the DNN can learn the metric and the mass simultaneously. In addition to various extensions, there are two open questions which should be mentioned. (i) Is there a better ansatz for the regularization term? Note that the regularization in this work is not to prevent overfitting as usual in machine learning. Instead, it is a guide to the minimum loss. We might need a deeper physical understanding of the regularization. (ii) How do we reduce the discretized error at high frequencies and low temperatures sufficiently? Compared with directly increasing the number of layers, a more efficient method might be

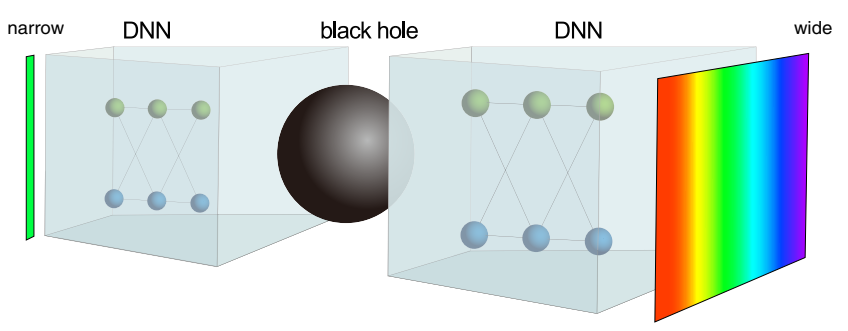

FIG. 4. Schematic diagram: the DNN encodes the black hole spacetime by which a wide spectrum of the field theory data can be generated from its narrow piece.

to apply the recently proposed DNN models of ordinary differential equations [64]. We ultimately hope that our work could suggest a data-driven way to study holographic transports.

Moreover, we found that the complete black hole metric from IR to UV can be well learned from the data with narrow frequency ranges. We also checked that the performance of the DNN is hardly changed by randomly deleting several data points in our numerical experiments. These two facts indicate that the shear viscosity encodes the spacetime in a very different way from the entanglement entropy. The latter probes the deeper spacetime only by the $S(l)$ with a larger $l$, so any data point is necessary to reconstruct the spacetime. Perhaps we can describe the difference concisely as follows: The nonlocal observable (entanglement entropy) on the boundary probes the bulk spacetime locally, while the local observable (shear viscosity) probes it nonlocally. Furthermore, this nonlocality leads to the excellent generalization ability of the DNN, which should be important in the application to the experimental data collected only in a part of the spectrum. Theoretically, from the perspective of machine learning, it usually implies that the data are highly structured. ${ }^{6}$ This structure is often important but obscure ${ }^{7}$ due to the infamous black-box problem of machine learning. However, here the structure is nothing but the higherdimensional black hole spacetime. This strong emergence might shed some light on the understanding of DL. Last but not least, the excellent generalization suggests that the strongly coupled field theories with a gravity dual could exhibit another feature of the hologram in addition to encoding the higher dimension: The local (a small piece of the hologram) can reproduce the whole; see the schematic diagram Fig. 4.

\footnotetext{
${ }^{6}$ Another possibility is that the network has some symmetry [65].

${ }^{7}$ For example, using the generative adversarial network, the approximate statistical predictions have been made recently in the string theory landscape [66], where the accurate extrapolation capability has been exhibited on simulating Kähler metrics. It was speculated that this is the first evidence of Reid's fantasy: All Calabi-Yau manifolds with fixed dimension are connected.
} 


\section{ACKNOWLEDGMENTS}

We thank Koji Hashimoto for reading the draft and giving valuable comments. We also thank Matteo Baggioli, Yongcheng Ding, Wei-Jia Li, Tomi Ohtsuki, and Fu-Wen Shu for helpful discussions. S. F. W. and X. H. G. are supported by National Natural Science Foundation of
China Grants No. 11675097 and No. 11875184, respectively. Y. T. is supported partially by NSFC Grants No. 11975235 and No. 11675015. He is also supported by the "Strategic Priority Research Program of the Chinese Academy of Sciences" with Grant No. XDB23030000.
[1] K. G. Wilson and J. Kogut, Phys. Rep. 12, 75 (1974).

[2] K. G. Wilson, Rev. Mod. Phys. 55, 583 (1983).

[3] J. Polchinski, Nucl. Phys. B231, 269 (1984).

[4] L. P. Kadanoff, Phys. Phys. Fiz. 2, 263 (1966).

[5] T. J. Sejnowski, The Deep Learning Revolution (MIT Press, Cambridge, MA, 2018).

[6] X. G. Wen, http://url.cn/5eKhBBP (in Chinese).

[7] P. Mehta and D. J. Schwab, arXiv:1410.3831.

[8] C. Bény, arXiv:1301.3124.

[9] S. Saremi and T. J. Sejnowski, Proc. Natl. Acad. Sci. U.S.A. 110, 3071 (2013).

[10] A. Paul and S. Venkatasubramanian, arXiv:1412.6621.

[11] S. Braddea and W. Bialek, J. Stat. Phys. 167, 462 (2017).

[12] D. Oprisa and P. Toth, arXiv:1705.11023.

[13] S. Foreman, J. Giedt, Y. Meurice, and J. Unmuth-Yockey, Eur. Phys. J. Web Conf. 175, 11025 (2018).

[14] S. Iso, S. Shiba, and S. Yokoo, Phys. Rev. E 97, 053304 (2018).

[15] S. H. Li and L. Wang, Phys. Rev. Lett. 121, 260601 (2018).

[16] H. W. Lin, M. Tegmark, and D. Rolnick, J. Stat. Phys. 168, 1223 (2017).

[17] M. Koch-Janusz and Z. Ringel, Nat. Phys. 14, 578 (2018); P. M. Lenggenhager, D. E. Gokmen, Z. Ringel, S. D. Huber, and M. Koch-Janusz, Phys. Rev. X 10, 011037 (2020).

[18] J. M. Maldacena, Adv. Theor. Math. Phys. 2, 231 (1998).

[19] S. S. Gubser, I. R. Klebanov, and A. M. Polyakov, Phys. Lett. B 428, 105 (1998).

[20] E. Witten, Adv. Theor. Math. Phys. 2, 253 (1998).

[21] L. Susskind and E. Witten, arXiv:hep-th/9805114.

[22] J. C. Bridgeman and C. T. Chubb, J. Phys. A 50, 223001 (2017).

[23] U. Schollwöck, Ann. Phys. (Amsterdam) 326, 96 (2011).

[24] G. Evenbly and G. Vidal, J. Stat. Phys. 145, 891 (2011).

[25] G. Vidal, Phys. Rev. Lett. 99, 220405 (2007); 101, 110501 (2008); G. Evenbly and G. Vidal, Phys. Rev. B 79, 144108 (2009).

[26] W. C. Gan and F. W. Shu, Int. J. Mod. Phys. D 26, 1743020 (2017).

[27] Y.-Z. You, Z. Yang, and X.-L. Qi, Phys. Rev. B 97, 045153 (2018).

[28] K. Hashimoto, S. Sugishita, A. Tanaka, and A. Tomiya, Phys. Rev. D 98, 046019 (2018).

[29] K. Hashimoto, S. Sugishita, A. Tanaka, and A. Tomiya, Phys. Rev. D 98, 106014 (2018).

[30] K. Hashimoto, Phys. Rev. D 99, 106017 (2019).

[31] H. Y. Hu, S. H. Li, L. Wang, and Y.Z. You, Phys. Rev. Research 2, 023369 (2020).
[32] X. Han and S. A. Hartnoll, Phys. Rev. X 10, 011069 (2020).

[33] J. Tan and C. B. Chen, Int. J. Mod. Phys. D 28, 1950153 (2019).

[34] J. Zaanen, Y. W. Sun, and Y. Liu, Holographic Duality in Condensed Matter Physics (Cambridge University Press, Cambridge, England, 2015), Sec. VII.

[35] P. K. Kovtun, D. T. Son, and A. O. Starinets, Phys. Rev. Lett. 94, 111601 (2005).

[36] T. Schaefer and D. Teaney, Rep. Prog. Phys. 72, 126001 (2009).

[37] N. Iqbal and H. Liu, Phys. Rev. D 79, 025023 (2009).

[38] I. Bredberg, C. Keeler, V. Lysov, and A. Strominger, J. High Energy Phys. 03 (2011) 141.

[39] K. S. Thorne, R. H. Price, and D. A. Macdonald, Black Holes: The Membrane Paradigm (Yale University Press, New Haven, CT, 1986).

[40] M. K. Parikh and F. Wilczek, Phys. Rev. D 58, 064011 (1998).

[41] S. A. Hartnoll, D. M. Ramirez, and J. E. Santos, J. High Energy Phys. 03 (2016) 170.

[42] D. Nickel and D. T. Son, New J. Phys. 13, 075010 (2011).

[43] I. Heemskerk and J. Polchinski, J. High Energy Phys. 06 (2011) 031.

[44] T. Faulkner, H. Liu, and M. Rangamani, J. High Energy Phys. 08 (2011) 051.

[45] See Supplemental Material at http://link.aps.org/ supplemental/10.1103/PhysRevD.102.101902 for the derivation of the relation between the shear response and the shear viscosity, the details of the Einstein-Maxell-dilaton theory, and the training scheme and report.

[46] B. Bradlyn, M. Goldstein, and N. Read, Phys. Rev. B 86, 245309 (2012).

[47] M. Geracie, D. T. Son, C. Wu, and S. F. Wu, Phys. Rev. D 91, 045030 (2015).

[48] M. Henningson and K. Skenderis, J. High Energy Phys. 07 (1998) 023; S. de Haro, S. N. Solodukhin, and K. Skenderis, Commun. Math. Phys. 217, 595 (2001); M. Bianchi, D. Z. Freedman, and K. Skenderis, Nucl. Phys. B631, 159 (2002).

[49] J. de Boer, E. Verlinde, and H. Verlinde, J. High Energy Phys. 08 (2000) 003; D. Martelli and W. Mueck, Nucl. Phys. B654, 248 (2003); J. Kalkkinen, D. Martelli, and W. Mueck, J. High Energy Phys. 04 (2001) 036; I. Papadimitriou and K. Skenderis, IRMA Lect. Math. Theor. Phys. 8, 73 (2005); H. Elvang and M. Hadjiantonis, J. High Energy Phys. 06 (2016) 046; F. Chen, S. F. Wu, and Y. X. Peng, J. High Energy Phys. 07 (2019) 072.

[50] T. Jacobson, Classical Quantum Gravity 24, 5717 (2007). 
[51] T. Akutagawa, K. Hashimoto, and T. Sumimoto, Phys. Rev. D 102, 026020 (2020).

[52] T. Faulkner, H. Liu, J. McGreevy, and D. Vegh, Phys. Rev. D 83, 125002 (2011).

[53] M. Edalati, J. I. Jottar, and R. G. Leigh, J. High Energy Phys. 01 (2010) 018.

[54] T. Andrade, M. Baggioli, and O. Pujolàs, Phys. Rev. D 100, 106014 (2019).

[55] M. Baggioli, S. Grieninger, and H. Soltanpanahi, Phys. Rev. Lett. 124, 081601 (2020).

[56] S. S. Gubser and F. D. Rocha, Phys. Rev. D 81, 046001 (2010); K. Goldstein, S. Kachru, S. Prakash, and S. P. Trivedi, J. High Energy Phys. 08 (2010) 078; C. Charmousis, B. Gouteraux, B. S. Kim, E. Kiritsis, and R. Meyer, J. High Energy Phys. 11 (2010) 151.

[57] B. S. Kim, J. High Energy Phys. 11 (2016) 044.
[58] J. M. Maldacena, J. High Energy Phys. 04 (2003) 021.

[59] S. Ryu and T. Takayanagi, Phys. Rev. Lett. 96, 181602 (2006).

[60] B. Swingle, Phys. Rev. D 86, 065007 (2012).

[61] M. Van Raamsdonk, Gen. Relativ. Gravit. 42, 2323 (2010).

[62] J. Maldacena and L. Susskind, Fortschr. Phys. 61, 781 (2013).

[63] S. Bilson, J. High Energy Phys. 08 (2008) 073; 02 (2011) 050.

[64] R. T. Q. Chen., Y. Rubanova, J. Bettencourt, and D. Duvenaud, arXiv:1806.07366.

[65] For example, see C. Wang, H. Zhai, and Y. Z. You, Sci. Bull. 64, 1228 (2019).

[66] J. Halverson and C. Long, Fortschr. Phys. 68, 2000005 (2020). 